\title{
The Stability of a Procedure for the Recovery of Lost Samples in Band-Limited Signals
}

\author{
Paulo Jorge S. G. Ferreira
}

\begin{abstract}
In this paper we study the eigenvalues of a matrix $S$ which arises in the recovery of lost samples from oversampled band-limited signals. Emphasis is placed on the variation of the eigenvalues as a function of the distribution of the missing samples and as a function of the oversampling parameter. We present a number of results which help to understand the numerical difficulties that may occur in this class of problems, and ways to circumvent them.
\end{abstract}

Keywords - Sampling, lost samples, interpolation, eigenvalues, stability, ill-posed problems, band-limited signals.

\section{INTRODUCTION}

A band-limited signal is a function $f$ which belongs to the space $L_{2}$ of square-integrable functions and which has a Fourier transform $\hat{f}$

$$
\hat{f}(\omega)=\frac{1}{\sqrt{2 \pi}} \int_{-\infty}^{+\infty} f(t) e^{-j \omega t} d t
$$

such that

$$
f(t)=\frac{1}{\sqrt{2 \pi}} \int_{-\sigma}^{+\sigma} \hat{f}(\omega) e^{j \omega t} d \omega .
$$

The set of all such $f \in L_{2}$ will be denoted by $B_{2}(\sigma)$. By the Whittaker-Kotelnikov-Shannon sampling theorem [1, 2], any $f \in B_{2}(\sigma)$ can be reconstructed from samples $f(n T)$ $(-\infty<n<\infty)$ taken $T$ seconds apart if $T \leq \pi / \sigma$.

When the oversampling parameter $r=\sigma T / \pi$ is less than unity, the samples $\{f(n T)\}$ are redundant, that is, any finite number of them can be obtained from the remaining ones by solving a system of linear equations [2-4]. This follows from the (absolutely and uniformly convergent) sampling series for $f \in B_{2}(\sigma)$,

$$
\begin{aligned}
f(t) & =\sum_{k=-\infty}^{+\infty} f(k T) \frac{\sin [\sigma(t-k T)}{\Omega(t-k T)} \\
& =r \sum_{k=-\infty}^{+\infty} f(k T) \operatorname{sinc}\left[r\left(\frac{t}{T}-k\right)\right],
\end{aligned}
$$

where $\operatorname{sinc}(x)=\sin (\pi x) / \pi x$. Assume that $n$ samples $f\left(i_{k} T\right)(k=0,1, \ldots, n-1)$ are unknown, and let $U=$ $\left\{i_{0}, i_{1}, \ldots, i_{n-1}\right\}$. Setting $t=i_{k} T$ in (1) we obtain

$$
\mathbf{u}=\mathbf{S u}+\mathbf{h},
$$

The author is with Departamento de Electrónica e Telecomunicações, Universidade de Aveiro, 3800 Aveiro, Portugal. where $\mathbf{u}$ is the $n$-dimensional vector of unknown samples $f\left(i_{k} T\right), \mathbf{S}$ is a matrix with elements

$$
S_{j k}=r \operatorname{sinc}\left[r\left(i_{j}-i_{k}\right)\right],
$$

and $\mathbf{h}$ is a known $n$-dimensional vector given by

$$
h_{j}=r \sum_{k \notin U} f(k T) \operatorname{sinc}\left[r\left(i_{j}-k\right)\right] .
$$

The band-limited signal can be determined if (2) can be solved for $\mathbf{u}$. It is easy to show that $\mathbf{S}$ is positive definite, independently of the number and distribution of the lost samples. Theoretically, this guarantees the existence and uniqueness of the solution to the reconstruction problem, but, in practice, a number of serious difficulties may occur. In this paper we present a number of results which clarify the eigenstructure of $\mathbf{S}$ and help in understanding and circumventing those problems.

\section{SOME PRACTICAL CONSIDERATIONS}

The positive definite character of $\mathbf{S}$ is not sufficient to ensure that (2) can be solved. In fact, if one of the eigenvalues of $\mathbf{S}$ is close to unity $\mathbf{I}-\mathbf{S}$ will be ill-conditioned, and its inversion may become an impossible task.

It can be shown that the largest eigenvalue of $\mathbf{S}$ is less than unity. This does not ensure that $\mathbf{I}-\mathbf{S}$ is wellconditioned, but it does suggest the use of iterative techniques as an alternative to direct inversion of $\mathbf{I}-\mathbf{S}$. Simple iteration of (2), for example, theoretically generates a converging sequence which approximates the solution. Unfortunately, this and a number of other iterative methods are also of limited usefulness when the largest eigenvalue of $\mathbf{S}$ approaches unity, due to the very poor convergence rates that might result.

In addition to these possible difficulties, the matrix $\mathbf{S}$ does not seem to possess any properties which render the solution computationally less expensive or more exact (although $\mathbf{S}$ will be Toeplitz if the lost samples are equidis$\operatorname{tant})$.

These considerations are important for some applications, including the transmission or storage of sampled data. The presence of burst errors will generally lead to an interpolation problem very ill-posed, and may result in large estimation errors if the values of the known samples 


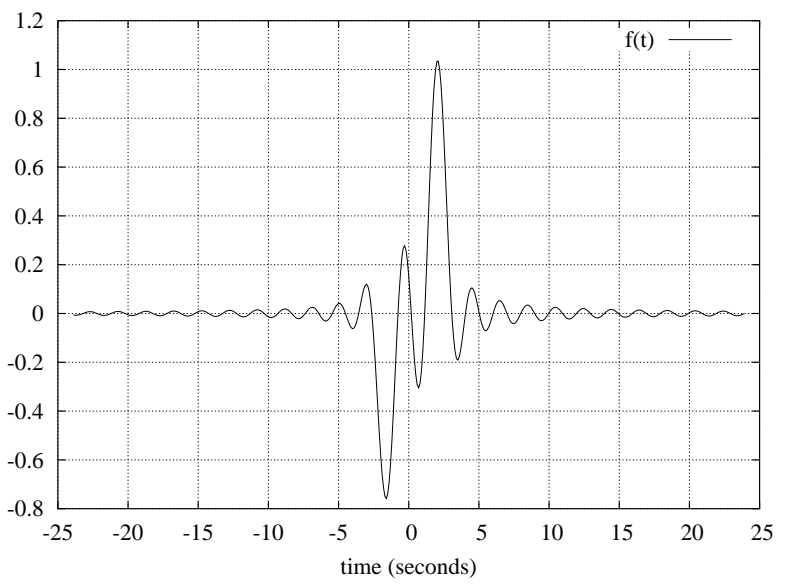

Fig. 1. Band-limited function $f(t)$.

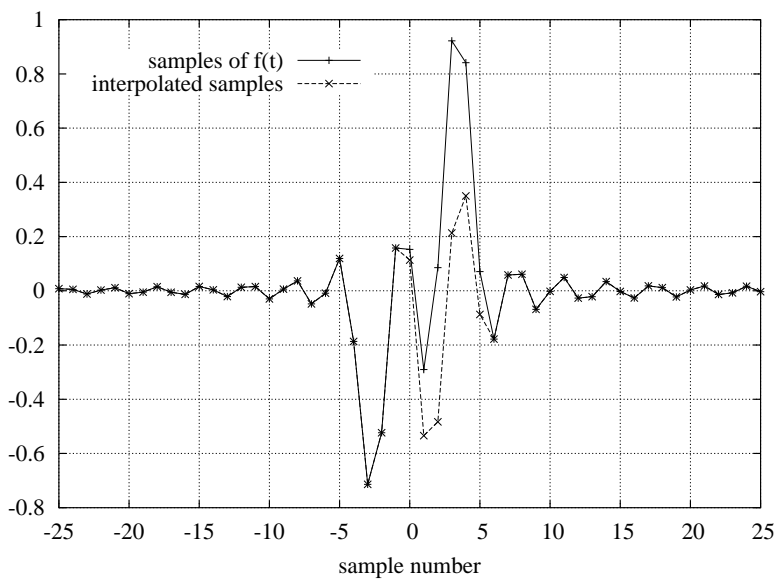

Fig. 2. Original and interpolated samples of $f(t)$. Contiguous lost samples: $f(0)$ through $f(5 T)$.

are not exactly known. Also, quantities such as $\mathbf{h}$ in (2) cannot be accurately computed since they depend on an infinite number of samples, and, in practice, only a finite number will be available.

To illustrate these facts, and motivate the study which will be subsequently carried out, we will present a few simple examples. Let

$$
f(t)=\operatorname{sinc}(t-2.1)-0.7 \operatorname{sinc}(t+1.7)
$$

be sampled with $r=0.6$. A segment of the function $f$ is depicted in figure 1. Assume that samples $f(n T)$, with $-40 \leq n \leq 40$, are all available for processing with the exception of 6 consecutive samples starting with $f(0)$. Despite the very low number of unknown samples, their interpolation based on (2) gives useless results (see figure 2). Compare this with figure 3 , which depicts the results of a similar experiment, but in which there were 30 missing samples more evenly spread apart (the randomly chosen sampling set is depicted in figure 4).

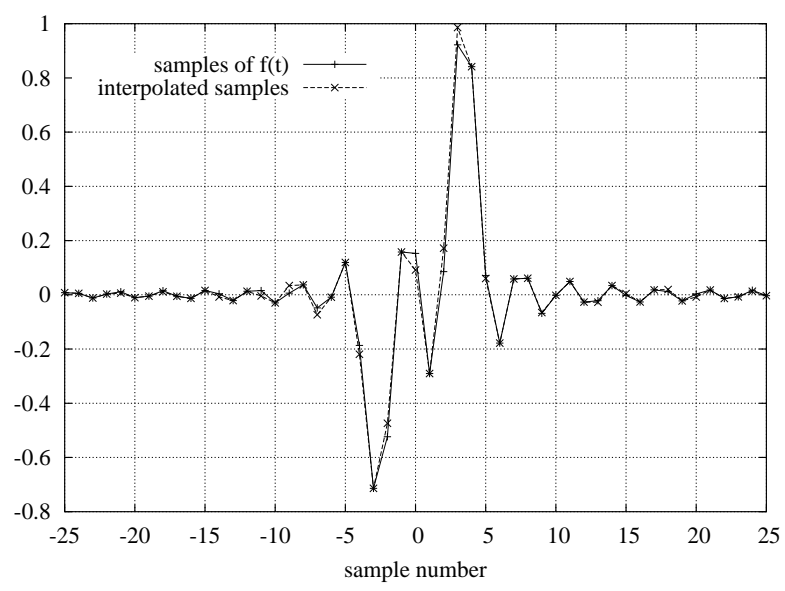

Fig. 3. Original and interpolated samples of $f(t)$. Thirty noncontiguous lost samples.

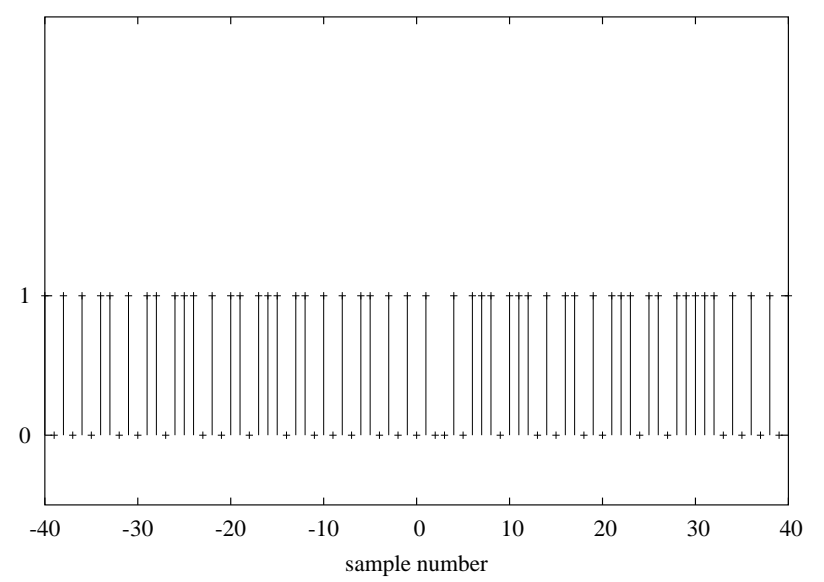

Fig. 4. Sampling set used in the interpolation problem depicted in figure 3.

These performance differences are due to the dependence of the eigenvalues of $\mathbf{S}$ on the positions of the lost samples. Figure 5 depicts the largest eigenvalue of $\mathbf{S}$ for several values of $r$, as a function of the matrix order $n$ (the number of unknown samples), assuming that they are contiguous, that is, $U=\{0,1, \ldots, n-1\}$. It clearly shows how close to unity the largest eigenvalue of $\mathbf{S}$ may get, even when the number of missing samples is reduced (check $r=0.6$ and $n=6$, for example). An interesting study of the effects of truncation and noise on the interpolation of burst errors in band-limited discrete signals may be found in [5].

One curious example of the dependence of the eigenvalues of $\mathbf{S}$ upon the distribution of the lost samples is given in figure 6 , which again depicts the largest eigenvalue of $\mathbf{S}$ for several values of $r$, as a function of the number of missing samples, but assuming that $U=\{0,4, \ldots, 4 n-4\}$. Examination of the results suggests that the maximum eigenvalue of $\mathbf{S}$ is bounded above by the smallest multiple of 


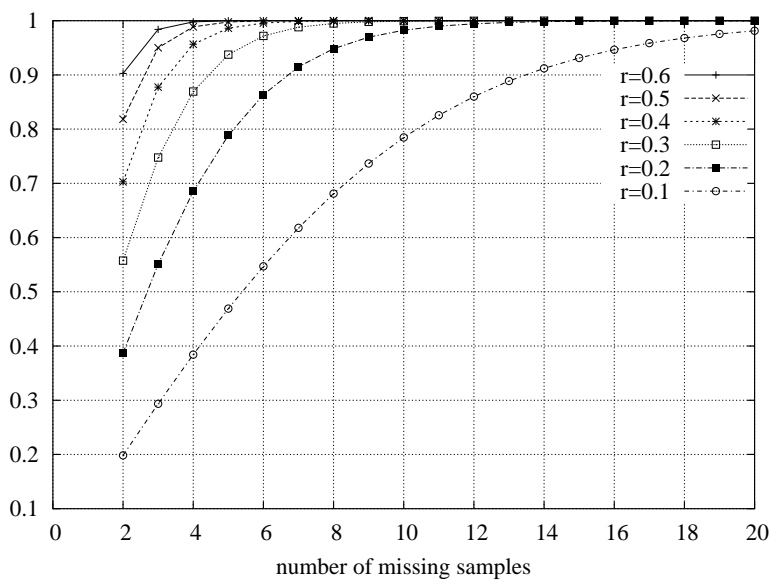

Fig. 5. Largest eigenvalue of $\mathbf{S} \in M_{n}$ as a function of $n$, for $U=\{0,1, \ldots, n-1\}$ and several possible values of $r$.

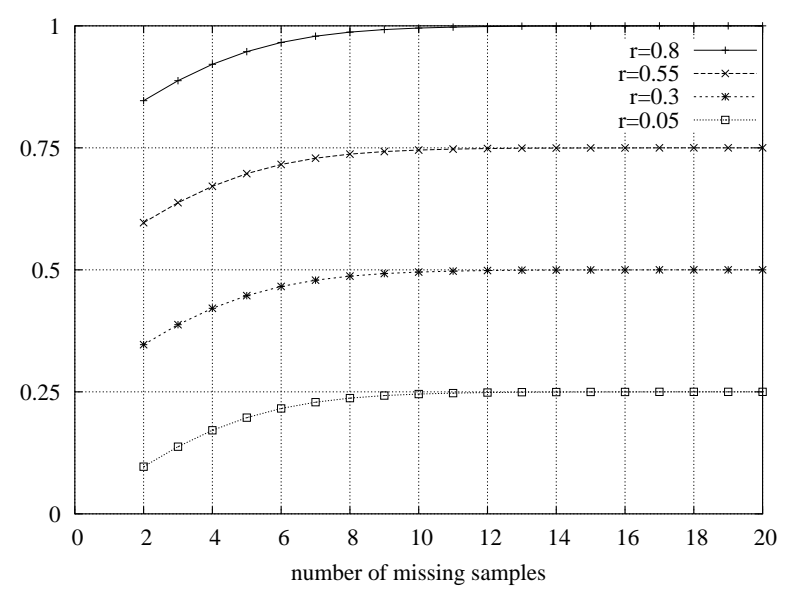

Fig. 6. Largest eigenvalue of $\mathbf{S} \in M_{n}$ as a function of $n$, for $U=\{0,4,8, \ldots, 4 n-4\}$, and several possible values of $r$.

$1 / 4$ greater than $r$, and below by the largest multiple of $1 / 4$ smaller than $r$, independently of the number of lost samples.

We will see that this is indeed true, even under more general conditions. If the positions of the missing samples are adequately chosen, the upper and lower bounds to the eigenvalues of $\mathbf{S}$ can be brought to values close to $r$ or a multiple of $r$, independently of the number of missing samples. This is a substantial improvement, and defines, as we will see, a subclass of well-posed data reconstruction problems which can be effectively solved using standard iterative or non-iterative techniques.

Our results explain the eigenstructure of $\mathbf{S}$ as a function of $r$ and $U$. The simplest results concern equidistant lost samples, that is, sets $U$ which contain only numbers of the form $u_{k}=k m$, for some fixed integer $m \geq 1$. It turns out that these results also apply if $u_{k}=i_{k} m$, for arbitrary integers $i_{k}$. We also address the recovery problem in its full generality, that is, when $U$ and $r$ are unrestricted.
Such a study may have important practical advantages. For example, our results suggest realistic interleaving factors for the samples of low-pass band-limited signals, prior to their transmission or archival. This interleaving would make the transmitted or stored information much less sensitive to burst errors, which would no longer tend to affect contiguous set of samples.

\section{The eigenvalues of $\mathbf{S}$}

We denote by $M_{n}$ the class of $n \times n$ matrices over the real or complex fields. The eigenvalues of a Hermitian matrix $\mathbf{A}$ are assumed to be sorted by increasing order $\lambda_{1} \leq$ $\lambda_{2} \leq \ldots \leq \lambda_{n}$. The spectral radius of $\mathbf{A}$, that is, its largest eigenvalue in absolute value, is denoted by $\rho(\mathbf{A})$. We are interested in the eigenvalues of $\mathbf{S} \in M_{n}$ defined by

$$
S_{j k}=r \operatorname{sinc}\left[r\left(i_{j}-i_{k}\right)\right],
$$

and their dependence on $r$ and $U=\left\{i_{0}, i_{1}, \ldots, i_{n-1}\right\}$. We write $\mathbf{S}(U), \mathbf{S}(r)$ or $\mathbf{S}(U, r)$ to emphasize the dependence of $\mathbf{S}$ on the generating set $U$ or on the parameter $r$. For example, $\mathbf{S}(\{0,4\}, 0.2)$ denotes the interpolation matrix which arises in the recovery of samples $f(0)$ and $f(4 T)$ of a band-limited signal $f$, oversampled with $r=0.2$. Note that it is possible to assume without loss of generality that $i_{0}=0$.

Clearly, $\mathbf{S}$ is a principal submatrix of the Toeplitz matrix $\mathbf{M}$ with elements

$$
M_{i k}=r \operatorname{sinc}[r(i-k)],
$$

where $i, k \in\left[0, i_{n-1}\right]$, and $0<r<1$. This matrix arises in the recovery of $i_{n-1}+1$ consecutive samples of $f(t)$, starting with sample zero, that is, $\mathbf{M}=\mathbf{S}\left(\left\{0,1, \ldots, i_{n-1}\right\}\right)$. However, $\mathbf{M}$ is also related to the discrete prolate spheroidal wave functions $[5,6]$.

The eigenvalues of $\mathbf{M}$ lie in the interval $(0,1)$. This follows from

$$
r \operatorname{sinc}(r x)=\frac{1}{2} \int_{-r}^{+r} e^{j \pi x y} d y
$$

which leads to

$$
\begin{aligned}
\mathbf{x}^{H} \mathbf{M x} & =\frac{1}{2} \sum_{k, \ell=0}^{i_{n-1}} x_{k} x_{\ell}^{*} \int_{-r}^{+r} e^{j \pi(k-\ell) y} d y \\
& =\frac{1}{2} \int_{-r}^{+r}\left|\sum_{k=0}^{i_{n-1}} x_{k} e^{j \pi k y}\right|^{2} d y>0
\end{aligned}
$$

if $\mathbf{x} \neq 0$. Thus, $\mathbf{M}$ is positive definite and its smallest eigenvalue greater than zero. That its largest eigenvalue is less than unity follows from

$$
\mathbf{x}^{H} \mathbf{M x}=\frac{1}{2} \int_{-r}^{+r}\left|\sum_{k=0}^{i_{n-1}} x_{k} e^{j \pi k y}\right|^{2} d y
$$




$$
\begin{aligned}
& <\frac{1}{2} \int_{-1}^{+1}\left|\sum_{k=0}^{i_{n-1}} x_{k} e^{j \pi k y}\right|^{2} d y \\
& =\sum_{k=0}^{i_{n-1}}\left|x_{k}\right|^{2}=\mathbf{x}^{H} \mathbf{x}
\end{aligned}
$$

and the fact that the largest eigenvalue of $\mathbf{M}$ equals the supremum of $\mathbf{x}^{H} \mathbf{M x}$ taken over all $\mathbf{x}$ with unitary norm.

These bounds are well-known [6]. Interestingly, they also hold for the matrices that arise in the recovery of unknown samples in generalized sampling expansions [4].

Since $\mathbf{S}$ is a principal submatrix of $\mathbf{M}$, its eigenvalues separate those of $\mathbf{M}$ [7]. Thus, they also belong to $(0,1)$, which means that $\mathbf{S}$ is also positive definite, and $\rho(\mathbf{S})<1$.

How do the eigenvalues of $\mathbf{S}$ depend on $U$ ? Among all principal submatrices of $\mathbf{M}$ with a given order, which ones have minimum spectral radius? If $n$ samples $\left\{f\left(i_{k} T\right)\right\}$ of $f \in B_{2}(\sigma)$ are lost during a certain time interval, how does the distribution of the lost samples affect the stability of the reconstruction procedures which follow from (2)? These are questions of considerable practical and theoretical interest, which we will now attempt to address.

We will begin by considering missing sample distributions $U=\left\{i_{0}, i_{1}, \ldots, i_{n-1}\right\}$ such that the least common multiple of the $1 / i_{k}\left(i_{k} \neq 0\right)$ is $1 / m$, where, clearly, $m \geq 1$. This means that $U$ contains only multiples of $m$, that is,

$$
U=\left\{i_{0} m, i_{1} m, \ldots, i_{n-1} m\right\},
$$

for a possibly different set of integers $i_{k}$. When $i_{k}=k$, the missing samples are equidistant.

Theorem 1: Let $U=\left\{i_{0} m, i_{1} m, \ldots, i_{n-1} m\right\}$. The eigenvalues $\lambda_{i}$ of $\mathbf{S}(U, r)$ are all equal to $r$ if and only if $r=\ell / m$ ( $\ell$ a positive integer). Otherwise, $\ell / m<\lambda_{i}<(\ell+1) / m$ for all $i$, where $\ell=\lfloor\mathrm{rm}\rfloor$.

Here, $\lfloor a\rfloor$ denotes the greatest integer less than or equal to $a$. Using (3) we have, for any $r$,

$$
\mathbf{x}^{H} \mathbf{S} \mathbf{x}=\frac{1}{2} \int_{-r}^{+r}|\phi(y)|^{2} d y,
$$

where

$$
\phi(y)=\sum_{k=0}^{n-1} x_{k} e^{j \pi m i_{k} y}
$$

has period $2 / m$. The stationary points of (4) as a function of $\mathbf{x}$, subject to the constraint $\|\mathbf{x}\|=1$, are the eigenvalues of $\mathbf{S}$. The interval $[-1,1]$ contains exactly $m$ subintervals of length $2 / m$ and the functions $e^{j \pi m i_{k} y}$ are orthogonal in $[-1,1]$. Thus,

$$
\frac{1}{2} \int_{-1 / m}^{+1 / m}|\phi(y)|^{2} d y=\frac{1}{2 m} \int_{-1}^{+1}|\phi(y)|^{2} d y
$$

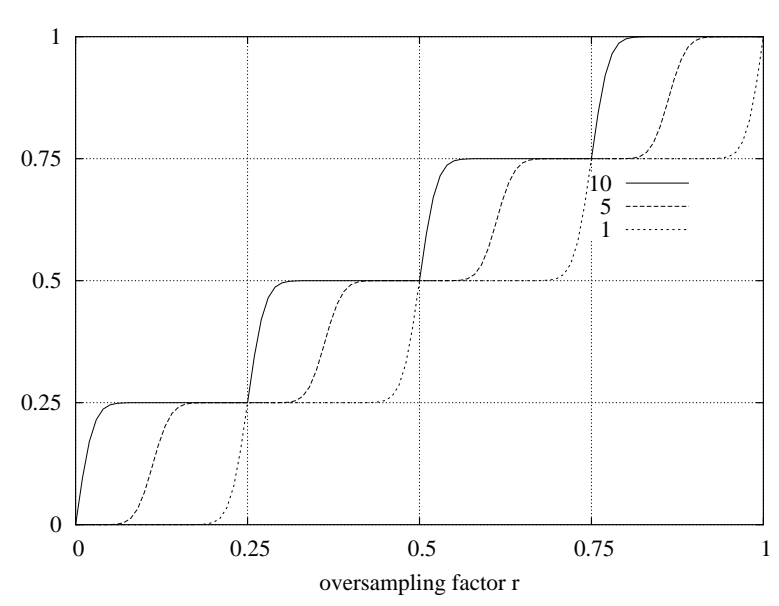

Fig. 7. Eigenvalues $\lambda_{1}$ (smallest), $\lambda_{5}$ and $\lambda_{10}$ (largest) of a $10 \times 10$ matrix $\mathbf{S}$ as a function of $r$, for $m=4$.

$$
\begin{aligned}
& =\left.\frac{1}{2 m} \int_{-1}^{+1}|| \sum_{k=0}^{n-1} x_{k} e^{j \pi m i_{k} y}\right|^{2} d y \\
& =\frac{1}{m} \sum_{k=0}^{n-1}\left|x_{k}\right|^{2}=\frac{1}{m},
\end{aligned}
$$

since $\|\mathbf{x}\|=1$. Now, if $0<r<1$ is not a multiple of $1 / m$, we have

$$
\begin{aligned}
\mathbf{x}^{H} \mathbf{S} \mathbf{x} & =\frac{1}{2} \int_{-r}^{+r}|\phi(y)|^{2} d y \\
& >\frac{\lfloor r m\rfloor}{2} \int_{-1 / m}^{+1 / m}|\phi(y)|^{2} d y \\
& =\frac{\lfloor r m\rfloor}{m},
\end{aligned}
$$

since $\lfloor r m\rfloor<m$. On the other hand,

$$
\begin{aligned}
\mathbf{x}^{H} \mathbf{S x} & =\frac{1}{2} \int_{-r}^{+r}|\phi(y)|^{2} d y \\
& <\frac{\lfloor r m\rfloor+1}{2} \int_{-1 / m}^{+1 / m}|\phi(y)|^{2} d y \\
& =\frac{\lfloor r m\rfloor+1}{m},
\end{aligned}
$$

since $\lfloor r m\rfloor+1>m r$. It remains to note that $\mathbf{S}=r \mathbf{I}$ if $r=$ $\ell / m, \ell$ any positive integer.

Theorem 1 explains figure 7, which depicts a few eigenvalues of $\mathbf{S}$ as a function of $r$, when $U=\{0,4,8, \ldots, 4 n-$ $4\}$. The bounds given in the theorem are independent of $n$ and extremely simple to calculate. Yet, their accuracy, which improves as $m$ increases, is already sufficient for practical assessment of many sampling sets $U$. A few examples are presented in table 1.

These bounds can always be combined with the following simple but useful result, valid for any distribution of missing samples. 
TABLE I

SMALLEST AND LARGEST EIGENVALUES OF $\mathbf{S}$ WHEN $U=\{0,8,12,40\}$, AND THEIR UPPER AND LOWER BOUNDS ACCORDING TO THEOREM 1.

\begin{tabular}{|c|ll|cc|}
\hline \hline$r$ & lower bound & upper bound & $\lambda_{1}$ & $\lambda_{4}$ \\
\hline 0.1 & 0 & 0.25 & 0.0145 & 0.1761 \\
0.2 & 0 & 0.25 & 0.1241 & 0.2496 \\
0.3 & 0.25 & 0.5 & 0.2504 & 0.3759 \\
0.4 & 0.25 & 0.5 & 0.3239 & 0.4856 \\
0.6 & 0.5 & 0.75 & 0.5145 & 0.6761 \\
0.7 & 0.5 & 0.75 & 0.6241 & 0.7496 \\
0.8 & 0.75 & 1 & 0.7504 & 0.8759 \\
0.9 & 0.75 & 1 & 0.8239 & 0.9855 \\
\hline
\end{tabular}

Theorem 2: Let $\lambda_{\min }$ and $\lambda_{\max }$ denote the minimum and maximum eigenvalues of $\mathbf{S}(r)$, respectively. Then, $\lambda_{\min } \leq$ $r$ and $\lambda_{\max } \geq r$, with equality if and only if $\mathbf{S}(r)$ is diagonal.

The trace of $\mathbf{S} \in M_{n}$ is $n r$, and equals the sum of the eigenvalues. But this means that

$$
n \lambda_{\min } \leq \sum_{i} \lambda_{i}=n r \leq n \lambda_{\max },
$$

which is the stated result.

Theorem 3: Let $\delta>0$ and $U=\left\{i_{0}, i_{1}, \ldots, i_{n-1}\right\}$. The minimum eigenvalue of $\mathbf{S}(r+\delta) \in M_{n}$ is not less than the minimum eigenvalue of $\mathbf{S}(r)$, and the maximum eigenvalue of $\mathbf{S}(r+\delta)$ does not exceed the maximum eigenvalue of $\mathbf{S}(r)$ by more than $n \delta$.

The theorem follows from the expression for the quadratic form

$$
\mathbf{x}^{H} \mathbf{S}(r+\delta) \mathbf{x}=\frac{1}{2} \int_{-r-\delta}^{r+\delta}\left|\sum_{k=0}^{n-1} x_{k} e^{j \pi i_{k} y}\right|^{2} d y,
$$

which implies

$$
\begin{aligned}
& \mathbf{x}^{H} \mathbf{S}(r+\delta) \mathbf{x}=\mathbf{x}^{H} \mathbf{S}(r) \mathbf{x} \\
& \quad+\frac{1}{2}\left(\int_{-r-\delta}^{-r}+\int_{r}^{r+\delta}\right)\left|\sum_{k=0}^{n-1} x_{k} e^{j \pi i_{k} y}\right|^{2} d y .
\end{aligned}
$$

Since the integrands are positive,

$$
\inf _{\|x\|=1} \mathbf{x}^{H} \mathbf{S}(r+\delta) \mathbf{x} \geq \inf _{\|x\|=1} \mathbf{x}^{H} \mathbf{S}(r) \mathbf{x},
$$

and therefore the minimum eigenvalue of $\mathbf{S}(r+\delta)$ cannot be less than that of $\mathbf{S}(r)$. On the other hand, the obvious upper bound to the integrals leads to

$$
\mathbf{x}^{H} \mathbf{S}(r+\delta) \mathbf{x} \leq \mathbf{x}^{H} \mathbf{S}(r) \mathbf{x}+\delta\left|\sum_{k=0}^{n-1}\right| x_{k}||^{2} .
$$

But the square of the maximum of $\sum_{k=0}^{n-1}\left|x_{k}\right|$, subject to the constraint $\|\mathbf{x}\|=1$, is $n$, and the stated result follows after considering the supremum over $\|x\|=1$ of each side of the equation.

If $r$ is rational $(r=p / q)$, placing the missing samples multiples of $q$ samples apart from each other, that is, letting

$$
U=\left\{i_{0} m, i_{1} m, \ldots, i_{n-1} m\right\},
$$

with $m=q$, brings $\mathbf{S}(U, r)$ to diagonal form $r \mathbf{I}$. This is the smallest value of $m$ for which this happens. If $r$ is irrational, it is still possible to find $m$ such that all eigenvalues of $\mathbf{S}$ lie as close to $r$ as we wish. This is a consequence of theorem 1, which shows that the mappings $f_{i}: r \rightarrow \lambda_{i}[\mathbf{S}(r)]$ tend to the unity map, uniformly, when $m \rightarrow \infty$. The following theorem makes this statement precise, and gives the minimum necessary value of $m$.

Theorem 4: Let $0<r<1$. For any prescribed $\varepsilon>0$, all eigenvalues of $\mathbf{S}(U, r) \in M_{n}$ will lie in $[r-\varepsilon, r+\varepsilon]$ if we choose

$$
m \geq \sqrt{\frac{n-1}{5 \varepsilon}},
$$

and set $U=\left\{i_{0} m, i_{1} m, \ldots, i_{n-1} m\right\}$.

By Hurwitz's theorem [8], any irrational $r$ has an infinity of irreducible rational approximations $p / q$ such that

$$
r=\frac{p}{q}+\frac{\theta}{\sqrt{5} q^{2}}
$$

where $0<|\theta|<1$, and the constant $\sqrt{5}$ is best-possible. We will assume that $\theta>0$, there being no essential changes if the opposite is true. Let $r^{\prime}=p / q$, and set $m$ equal to a multiple of $q$. The matrix $\mathbf{S}\left(U, r^{\prime}\right)$, where

$$
U=\left\{i_{0} m, i_{1} m, \ldots, i_{n-1} m\right\},
$$

is equal to $r^{\prime} \mathbf{I}$. Applying theorem 3 with

$$
\delta=\frac{\theta}{\sqrt{5} q^{2}}
$$

we find that all eigenvalues of $\mathbf{S}(U, r)$ must belong to

$$
\left[r^{\prime}, r^{\prime}+\frac{n \theta}{\sqrt{5} q^{2}}\right] \subset[r-\delta, r+(n-1) \delta] .
$$

Since $\delta$ is arbitrary, this shows that there is an $m$ such that all eigenvalues of $\mathbf{S}$ lie in $[r-\varepsilon, r+\varepsilon]$, for any prescribed 


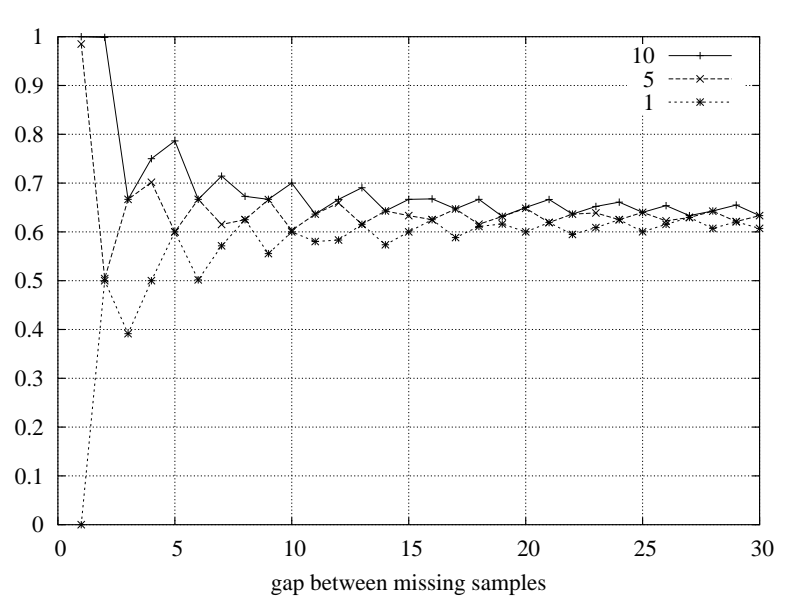

Fig. 8. Eigenvalues $\lambda_{1}$ (smallest), $\lambda_{5}$ and $\lambda_{10}$ (largest) of a $10 \times 10$ matrix $\mathbf{S}$ as a function of $m$, for $r=0.63$.

$\varepsilon>0$. Letting $\varepsilon=(n-1) /\left(\sqrt{5} q^{2}\right)$ and solving for $q$ gives the lower bound to $m$.

This result shows that proper selection of the gap between missing samples can bring the spectral radius of $\mathbf{S}$ to values arbitrarily near $r$. This is illustrated in figure 8, which shows some of the eigenvalues of $\mathbf{S}(U, r)$, for

$$
U=\left\{i_{0} m, i_{1} m, \ldots, i_{n-1} m\right\},
$$

and $r=0.63$, as a function of $m$.

Theorems 1 and 4 are restricted to

$$
U=\left\{i_{0} m, i_{1} m, \ldots, i_{n-1} m\right\} .
$$

In particular, if $i_{k}=k$, they apply to equidistant sets of lost samples. We will now study the more general case where the elements of $U$ are arbitrary distinct integers sorted by increasing order.

Examination of the form of $\mathbf{S}$ when one more element is added to the set $U$ immediately shows that the eigenvalues of the $n \times n$ matrix separate those of the larger. This is a direct consequence of the form of $\mathbf{S}$ and of the interlacing theorems for symmetric or bordered matrices [7]. This observation suggests that a study of the eigenvalues of $\mathbf{S}_{n}=\mathbf{S}\left(U_{n}\right)$ and $\mathbf{S}_{n+1}=\mathbf{S}\left(U_{n+1}\right)$ might be useful, where

$$
\begin{aligned}
U_{n} & =\left\{i_{0}, i_{1}, \ldots, i_{n-1}\right\}, \\
U_{n+1} & =\left\{i_{0}, i_{1}, \ldots, i_{n-1}, i_{n}\right\} .
\end{aligned}
$$

The following theorem is a consequence of this approach.

Theorem 5: Consider $\mathbf{S}_{n} \in M_{n}$ and $\mathbf{S}_{n+1} \in M_{n+1}$. If the eigenvalues $\lambda_{i}^{(n+1)}$ of $\mathbf{S}_{n+1}$ are arranged in increasing order, then

$$
\mu_{i}^{(n+1)}-c_{n} \leq \lambda_{i}^{(n+1)} \leq \mu_{i}^{(n+1)}+c_{n},
$$

where $\mu_{i}^{(n+1)}$ is the sequence of eigenvalues of $\mathbf{S}_{n}$ together with $r$, sorted by increasing order, and

$$
c_{n}^{2}=r^{2} \sum_{k=0}^{n-1} \operatorname{sinc}^{2}\left[r\left(i_{n}-i_{k}\right)\right] .
$$

The matrix $\mathbf{S}_{n+1} \in M_{n+1}$ can be written in partitioned form as

$$
\mathbf{S}_{n+1}=\left[\begin{array}{c|c}
\mathbf{S}_{n} & \mathbf{a}_{n} \\
\hline \mathbf{a}_{n}^{T} & r
\end{array}\right],
$$

where the $k$-th element of the vector $\mathbf{a}_{n}$ is $r \operatorname{sinc}\left[r\left(i_{n}-i_{k}\right)\right]$, $k=0,1, \ldots, n-1$. We may express $\mathbf{S}_{n+1}$ as the sum of two real symmetric matrices

$$
\mathbf{S}_{n+1}=\left[\begin{array}{c|c}
\mathbf{S}_{n} & 0 \\
\hline 0 & r
\end{array}\right]+\left[\begin{array}{c|c}
0 & \mathbf{a}_{n} \\
\hline \mathbf{a}_{n}^{T} & 0
\end{array}\right] .
$$

The eigenvalues of the first of these two matrices are clearly those of $\mathbf{S}_{n}$ itself, plus $r$. When arranged by increasing order, these $n+1$ numbers form the sequence $\mu_{k}^{(n+1)}$. It is straightforward to show that the nonzero eigenvalues of the second matrix are the two square roots of

$$
\left\|\mathbf{a}_{n}\right\|^{2}=r^{2} \sum_{k=0}^{n-1} \operatorname{sinc}^{2}\left[r\left(i_{n}-i_{k}\right)\right] .
$$

By Weyl's theorem on the eigenvalues of the sum of two symmetric matrices [7] one has

$$
\mu_{k}^{(n+1)}-\left\|\mathbf{a}_{n}\right\| \leq \lambda_{k}^{(n+1)} \leq \mu_{k}^{(n+1)}+\left\|\mathbf{a}_{n}\right\|,
$$

and the theorem follows if we define $c_{n}=\left\|\mathbf{a}_{n}\right\|$.

The following simple bound to the constants $c_{n}$, given in terms of the minimum spacing between missing samples, is sometimes useful.

Theorem 6: Let $\ell$ be the minimum separation between the elements of $U$, that is,

$$
\ell=\min _{i, j \in U}|i-j| .
$$

The constants $c_{n}$ in theorem 5 satisfy

$$
c_{n} \leq \frac{1}{\ell \sqrt{6}} \text {. }
$$

This is a consequence of

$$
\begin{aligned}
c_{n}^{2}=r^{2} \sum_{k=0}^{n-1} \operatorname{sinc}^{2}\left[r\left(i_{n}-i_{k}\right)\right] & \leq \frac{1}{\pi^{2}} \sum_{k=0}^{n-1} \frac{1}{\left(i_{n}-i_{k}\right)^{2}} \\
& \leq \frac{1}{\pi^{2}} \sum_{k=0}^{n-1} \frac{1}{\ell^{2}(n-k)^{2}} \\
& \leq \frac{1}{6 \ell^{2}},
\end{aligned}
$$


using Euler's classical result $\sum_{k>0} 1 / k^{2}=\pi^{2} / 6$.

These results show how the eigenvalues of $\mathbf{S}$ change when one missing sample is added to $U$. Consider the set $E_{n}$ of $n$ eigenvalues of $\mathbf{S}$, whose average value is clearly $r$. By theorem 2, the largest of these eigenvalues is greater than $r$, and the smallest less than $r$. They are all equal to $r$ if $\mathbf{S}$ is diagonal.

To obtain bounds for the eigenvalues of the matrix that results when one sample is added to $U$, consider the union $E_{n} \cup\{r\}$, and denote by $X_{n+1}$ the ordered set of $n+1$ numbers obtained. Their average, maximum and minimum values are obviously the same as those of $E_{n}$. The bounds for the eigenvalues of the new matrix are obtained by addition and subtraction of $c_{n}$ to each of the elements of $X_{n+1}$.

Note that $c_{n}$ can be zero if and only if $U$ contains only multiples of $m$, and $r$ is itself a multiple of $1 / \mathrm{m}$. In this case, all eigenvalues remain equal to $r$ and the matrix keeps its diagonal form.

By placing the missing samples sufficiently far apart, $c_{n}$ can be made as small as desired. The worst possible situation occurs when the missing samples are contiguous, in which case, as figure 5 shows, the spectral radius may grow rapidly with $n$.

It is now easy to find upper and lower bounds for the eigenvalues of $\mathbf{S}(U, r)$, under no restrictions on $U$ or $r$. For example, successive invocations of theorem 5 show that the eigenvalues of $\mathbf{S}(U, r) \in M_{n}$ are bounded by the numbers

$$
r \pm \sum_{i=1}^{n-1} c_{i}
$$

Using the bound given in theorem 6 this implies the weaker result

$$
r \pm \frac{n-1}{\ell \sqrt{6}}
$$

where, as before, $\ell$ is the minimum separation between elements of $U$.

\section{SOLVING THE RECONSTRUCTION PROBLEM}

The stability of the interpolation method expressed by (2) depends strongly on the set $U$, that is, on the missing sample distribution. The previous results clarify this dependence and help in understanding the practical problems that may occur.

Equation (2) may be solved using direct or iterative techniques. When the number of missing samples is large, or when the largest eigenvalue of $\mathbf{S}$ approaches unity, the iterative methods become valuable alternatives. To complete our discussion we will briefly mention a few such methods.
The simplest technique is direct iteration of (2), which leads to

$$
\mathbf{u}_{i+1}=\mathbf{S} \mathbf{u}_{i}+\mathbf{h} .
$$

Equation (2), in the form

$$
\mathbf{u}=\mu(\mathbf{S u}+\mathbf{h})+(1-\mu) \mathbf{u},
$$

also suggests the iterative method

$$
\begin{aligned}
\mathbf{u}_{i+1} & =\mu\left(\mathbf{S u}_{i}+\mathbf{h}\right)+(1-\mu) \mathbf{u}_{i} \\
& =[(1-\mu) \mathbf{I}+\mu \mathbf{S}] \mathbf{u}_{i}+\mu \mathbf{h},
\end{aligned}
$$

where the real $\mu$ can be adjusted to control the convergence rate. When $\mu=1$ this reduces to (5).

Let $\mathbf{T}=\mathbf{I}-\mathbf{S}$. Expressing $\mathbf{T}$ as $\mathbf{T}=\mathbf{M}-\mathbf{N}$ leads to yet another alternative form for (2),

$$
\mathbf{u}=\mathbf{M}^{-1} \mathbf{N u}+\mathbf{M}^{-1} \mathbf{h} .
$$

Different choices for $\mathbf{M}$ and $\mathbf{N}$ lead to different iterative methods.

Let $\mathbf{D}, \mathbf{L}$ and $\mathbf{U}$ denote diagonal, lower triangular, and upper triangular matrices, such that $\mathbf{T}=\mathbf{D}-\mathbf{L}-\mathbf{U}$. Assume that the main diagonal elements of $\mathbf{D}$ equal those of $\mathbf{T}$. Thus, the main diagonals of $\mathbf{L}$ and $\mathbf{U}$ contain only zero elements, and their nonzero elements differ from the corresponding elements of $\mathbf{T}$ only in sign.

Letting $\mathbf{M}=\mathbf{D}$ leads to Jacobi's iteration,

$$
\mathbf{u}_{i+1}=\mathbf{D}^{-1}(\mathbf{L}+\mathbf{U}) \mathbf{u}_{i}+\mathbf{D}^{-1} \mathbf{h} .
$$

whereas $\mathbf{M}=\mathbf{D}-\mathbf{L}$ yields the Gauss-Seidel iteration,

$$
\mathbf{u}_{i+1}=(\mathbf{D}-\mathbf{L})^{-1} \mathbf{U} \mathbf{u}_{i}+(\mathbf{D}-\mathbf{L})^{-1} \mathbf{h} .
$$

The Gauss-Seidel method can be implemented as Jacobi's method, but performing the computations necessary to find each approximation in-place. This avoids inversion of $\mathbf{D}-$ $\mathbf{L}$ and cuts the storage requirements somewhat.

A relaxation constant $\mu$ may be incorporated in both methods, leading to the JOR and SOR iterations. References $[9,10]$ contain detailed studies of these as well as other iterative methods for the solution of linear equations.

As a final remark, we note that it is possible to formulate a reconstruction problem similar to the one studied in this paper for finite-dimensional signals, that is, signals with a finite number of samples. These methods can also be used in that context, and sufficient conditions for their convergence have been given [11].

\section{Conclusions}

The results presented herein may have practical applications, some of which are intuitively apparent. For example, the transmission or archival of sampled data, over 
channels or on media where the probability of burst errors is high, might be best accomplished if the samples are interleaved before transmission/archival. If the interleaving factor is too large, there will be unnecessary delays and memory needs. Our results simplify the computation of reasonable interleaving factors, yet large enough to lead to a well-posed interpolation problem.

Even under ideal noiseless conditions, interpolation with the sampling series will never be exact due to the necessary truncation of the infinite series (1). Quantities such as $\mathbf{h}$ in (2), which depend on an infinite number of samples, can only be approximately found. As we have seen, the accumulated effect of truncation error and all other unavoidable errors, such as those introduced by finite-precision arithmetic, measurement error, etc., may be felt even in interpolation problems of moderate size. The magnitude of those effects, however, strongly depends on the oversampling parameter $r$ and the distribution of the missing samples. Our results help to quantitatively understand the effect of these factors.

The interpolation of band-limited low-pass signals in the presence of missing samples, although theoretically possible using (2), may become a very difficult numerical task if a number of contiguous samples is unavailable or have been contaminated by noise. In that case, the largest eigenvalue of the reconstruction matrix $\mathbf{S}$ in (2) may approach unity, leading to a very ill-posed problem. Stabilizing or windowing techniques such as the perturbation technique described in [5] might be useful in such cases, but the success of the reconstruction critically depends on the value of the oversampling parameter $r$ and on the number $n$ of missing or affected samples. In general, good results are possible only for low values of $r$ and $n$, that is, only when a few missing samples are missing, and/or the samples came from a highly oversampled signal.

If the unknown samples are not contiguous, the reconstruction problem is in general much more tractable, unless, of course, $r$ is very close to unity. Recall that the largest eigenvalue of the reconstruction matrix $\mathbf{S}$ is always larger than $r$ (theorem 2). Therefore, if $r$ is close to unity, that is, if the sampling frequency is very near its critical lowest possible value set by the sampling theorem, the reconstruction will be difficult regardless of the missing sample distribution.

When $r$ is not so close to unity, the reconstruction matrix $\mathbf{S}$ will normally have a reasonably low spectral radius, assuming that the gaps between missing samples are sufficiently large. Our results show that the largest eigenvalue of the reconstruction matrix $\mathbf{S}$ may reach values nearly as low as $r$, independently of the number of lost samples. In many cases, the interpolation can be performed safely, us- ing non-iterative methods (direct inversion of $\mathbf{I}-\mathbf{S}$ ) or one of the several iterative methods available, the simplest of which is direct iteration of equation (2). When the missing sample positions are all multiples of an integer $m>1$ and $r$ is an appropriate rational, $\mathbf{S}$ may even be diagonal, and the recovery process trivial.

We gave several useful bounds to the eigenvalues of $\mathbf{S}$, and examples which illustrate and confirm the main results. It is hoped that the preceding results might help in understanding some of the problems that arise when dealing with sampled data in a practical environment.

\section{REFERENCES}

[1] A. J. Jerri, "The Shannon sampling theorem - its various extensions and applications: a tutorial review", Proceedings of the IEEE, vol. 65, no. 11, pp. 1565-1596, Nov. 1977.

[2] R. J. Marks II, Introduction to Shannon Sampling and Interpolation Theory, Springer, Berlin, 1991.

[3] R. J. Marks II, "Restoring lost samples from an oversampled band-limited signal", IEEE Transactions on Acoustics, Speech, and Signal Processing, vol. 31, no. 3, pp. 752-755, June 1983.

[4] P. J. S. G. Ferreira, "Incomplete sampling series and the recovery of missing samples from oversampled band-limited signals", IEEE Transactions on Signal Processing, vol. 40, no. 1, pp. 225227, Jan. 1992.

[5] P. Delsarte, A. J. E. M. Janssen, and L. B. Vries, "Discrete prolate spheroidal wave functions and interpolation", SIAM Journal of Applied Mathematics, vol. 45, no. 4, pp. 641-650, Aug. 1985.

[6] D. Slepian, "Prolate spheroidal wave functions, Fourier analysis and uncertainty - V: The discrete case", The Bell System Technical Journal, vol. 57, no. 5, pp. 1371-1429, May 1978.

[7] R. A. Horn and C. R. Johnson, Matrix Analysis, Cambridge University Press, Cambridge, 1990.

[8] G. H. Hardy and E. M. Wright, An Introduction to the Theory of Numbers, Clarendon Press, Oxford, 1954.

[9] R. S. Varga, Matrix Iterative Analysis, Prentice-Hall, Englewood Cliffs, New Jersey, 1962

[10] D. M. Young, Iterative Solution of Large Linear Systems, Academic Press, Orlando, 1971.

[11] P. J. S. G. Ferreira, "Noniterative and faster iterative methods for interpolation and extrapolation", IEEE Transactions on Signal Processing, vol. 42, no. 11, pp. 3278-3282, Nov. 1994. 